\title{
Introduction to imaging methods in photosynthesis
}

\author{
Egbert J. Boekema
}

Received: 27 May 2009/Accepted: 10 August 2009/Published online: 8 September 2009

(c) The Author(s) 2009. This article is published with open access at Springerlink.com

Imaging is strongly coupled to microscopes. The first microscopes with a double lens system were built about 400 years ago by three Dutchmen, Cornelius Drebbel, Hans and Zacharias Jansen. Another Dutchman, Antoni van Leeuwenhoek, became famous somewhat later in the seventeenth century as the first experimental microscopist. He explored microorganisms with a simple microscope. Among his preserved specimens at the Royal Society in London are green algae and cotton seeds, to name a few topics related to photosynthesis (see: http://www.brianj ford.com/wavintr.htm).

Much later, in the nineteenth century, the German scientist Ernst Abbe formulated a famous mathematical theory correlating resolution to the wavelength of light. Abbe made clear that the maximum resolution in microscopes is fundamentally limited to roughly half of the applied wavelength. Because light microscopy depends on visible light of $\sim 400-700 \mathrm{~nm}$, the resolution of a light microscope is limited to about $200 \mathrm{~nm}(0.2 \mu \mathrm{m})$. Until recently, it turned out very hard to circumvent this so-called diffraction limit with light. Yet, in the 1930s of the last century, a side way with electrons was developed by Ernst Ruska. Electrons are particles but also have a wave character and can be accelerated to a speed close to the velocity of light. At an acceleration voltage of $100,000 \mathrm{~V}$ the wavelength of the electron beam is only $0.004 \mathrm{~nm}$. Ruska et al. managed in 1938 to construct an electron microscope that was already surpassing the resolution of the light microscope by a factor of 10. Since the early days the electron microscope has been gradually improved to an instrument which can achieve atomic resolution in the range of $0.05 \mathrm{~nm}$.

E. J. Boekema ( $\square)$

University of Groningen, Groningen, The Netherlands

e-mail: e.j.boekema@rug.nl
However, one should not get the impression that resolution is the only criterion for a biophysical technique. Imaging with a high energetic electron beam is actually in contrast to light microscopy a "single shot in the dark" because it quickly destroys the sample. Imaging with visible light, on the other hand, has the great advantage of being able to register dynamic processes. The development of three-dimensional light microscopy with confocal microscopes and the nowadays widespread application of in vivo fluorescent proteins, such as GFP, have been recognized as an important step in the development of science (see Nobel Prize for chemistry 2008 on nobelprize.org). This enabled ways to watch processes that were previously invisible, such as the development of nerve cells in the brain or how cancer cells spread. The recent increase in impact of (light) microscopy is also obvious by looking at the contributions in "Biophysical techniques in photosynthesis", a book with the same scope as this special issue, edited by the late Jan Amesz and Arnold Hoff in 1996 (Amesz and Hoff 1996). Of its 24 chapters, only one was devoted to (electron) microscopy.

Out of the many microscopy techniques, some traditional aspects and emerging methods relevant to photosynthesis have been selected for this part of the special issue. Four chapters are on light microscopy, two on EM, and one on scanning probe microscopy. In the first chapter, Cisek et al. start with a general introduction to light microscopy and its historical development. Emerging as well as most frequently used optical microscopy techniques are reviewed, including the above mentioned three-dimensional light microscopy with confocal microscopes and the enhancement of contrast by phase contrast microscopy. One of the emerging techniques is nonlinear microscopy. It presents numerous advantages over linear microscopy techniques including improved deep tissue imaging, optical sectioning, and imaging of live 
unstained samples. Nonetheless, nonlinear microscopy is in its infancy, lacking protocols, users, and applications; hence, this review focuses on the potential of nonlinear microscopy for studying photosynthetic organisms.

Fluorescence techniques have a special place in photosynthesis, not in the least because fluorescence provides information about the lifetime of the excited states. Chen and Clegg give a short account of lifetime-resolved imaging, in order to acquaint readers who are not experts with the basic methods for measuring lifetime-resolved signals throughout an image. They present the early fluorescence lifetime imaging (FLI) history, instruments and experiments and discuss briefly the fundamentals of the fluorescence response that one is measuring, and introduce the basic measurement methodologies. Fluorescence lifetime imaging microscopy (FLIM) is a technique that visualizes the excited state kinetics of fluorescence molecules with the spatial resolution of a fluorescence microscope. Measuring fluorescence lifetimes in many neighboring tiny volumes and displaying these in false colors as an image is the basis of FLIM. The size of the smallest measurement volume is limited by light diffraction; FLIM makes it therefore possible to image the heterogeneity of lifetimes within the spatial resolution of a light microscope. Petrášek et al. present a scanningless implementation of FLIM based on time- and space-correlated single photon counting (TSCSPC) method employing a position-sensitive quadrant anode detector and wide-field illumination. A third contribution to the topic of fluorescence is by Benediktyová and Nedbal (2009). Multi-color fluorescence emission from leaf tissues is presented as a powerful reporter on plant biochemistry and physiology. Mapping fluorescence along the leaf surface and also perpendicularly into the leaf depth becomes possible using novel macroscopic and microscopic imaging techniques. This contribution is focused on leaf fluorescence emission that is elicited by single-photon blue and red excitation and on the emission exited by simultaneous absorption of two infrared photons. With fluorescence microscopy leaf structures are visualized by red chlorophyll fluorescence emission reconstructed in three-dimensional images while the bacteria are detected by the green emission of engineered fluorescence protein.

EM has a long-term involvement in photosynthesis. The first important contributions came on the sub-cellular level when thin sectioning could reveal the ultrastructure of chloroplasts. Without EM it would have been difficult to understand basic phenomena such as the division in stacked and non-stacked photosynthetic (thylakoid) membranes. In the 1970s further insight was gained with freeze-fracturing and free-etching techniques. Staehelin (1976) showed, for instance, reversible particle movements associated with unstacking and restacking of these membranes. The freezefracturing and free-etching techniques have lost in popularity. The elaborative specimen preparation destroys the fine details, which is also the case in chemically fixed thin sections. Electron tomography is now state of the art in 3D EM, and is the topic of the presentation of HohmannMarriott and Roberson (2009). Much insight is to be gained by image processing because EM images are extremely noisy. In the last century, two processing lines became available, working either with two-dimensional crystals or with single particles. The latter has strongly gained in popularity and impact and is discussed by Boekema et al. (2009).

Besides light and electron microscopy, scanning probe microscopy (SPM) was developed in the 1980s as a third and very different way of performing microscopy. It is a technique to image surfaces using a physical probe that scans the specimen. An image of the surface is obtained by mechanically moving the probe in a raster scan of the specimen, line by line, and recording the probe-surface interaction as a function of position. Scanning tunneling microscope (STM) is the most sophisticated method in terms of resolution and works even well with single metal atoms. Atomic force microscopy (AFM) has turned out to be the most relevant for (membrane) proteins. Because it can be applied in aqueous solution, it has opened the way to follow in time the formation of protein arrays lipid bilayers (Reviakine et al. 1998). Although high quality AFM images are not easy to make in large numbers, they have a much lower noise level than EM images. Combined with a good resolution, this has enabled researchers to visualize, for instance, the small units in the rings of prokaryotic antenna complexes. This is one of the lasting contributions of this technique to the field of photosynthesis. Scheuring and Sturgis (2009) give an overview of AFM applied to the bacterial photosynthetic apparatus.

Last but not least, we have a contribution on nuclear magnetic resonance (NMR). NMR can be used in several ways, such as the characterization of small molecules from their spectra in organic chemistry. In the field of biophysics, its largest impact is on protein structure determination in solution. By the pioneering work of Kurt Wüthrich NMR became a useful technique in the 1980s to solve the structure of small protein molecules. One of the examples in photosynthesis is subunit $\mathrm{PsaC}$ from photosystem I (Antonkine et al. 2002). NMR can also be applied as an imaging tool, and magnetic resonance imaging (MRI) became a useful method in the same time. In its early years, the technique was referred to as nuclear magnetic resonance imaging. However, as the word nuclear was associated in the public mind with ionizing radiation exposure, the shorter abbreviation MRI became more popular. It provides on the scale of a human body a much greater contrast between the different soft tissues of the body than computed tomography with X-rays. Although 
MRI delivers a spatial resolution as good as a strong magnifying glass, it certainly delivers an abundant amount of information in addition to a reasonable spatial and temporal resolution. Part of this information, such as the flow of water in plant tissue, is very difficult to measure or cannot be measured using other techniques. This is the scope of the MRI paper of Van As et al. in the last contribution on imaging methods (Van As et al. 2009).

Open Access This article is distributed under the terms of the Creative Commons Attribution Noncommercial License which permits any noncommercial use, distribution, and reproduction in any medium, provided the original author(s) and source are credited.

\section{References}

Amesz J, Hoff AJ (eds) (1996) Biophysical techniques in photosynthesis. Kluwer Academic Publishers, Dordrecht

Antonkine ML, Liu G, Bentrop D, Bryant DA, Bertini I, Luchinat C, Golbeck JH, Stehlik D (2002) Solution structure of the unbound, oxidized Photosystem I subunit PsaC, containing [4Fe-4S] clusters $\mathrm{F}(\mathrm{A})$ and $\mathrm{F}(\mathrm{B})$ : a conformational change occurs upon binding to photosystem I. J Biol Inorg Chem 7:461-472
Benediktyová Z, Nedbal L (2009) Imaging of fluorescence emission from plant tissues. Photosynth Res (this issue)

Boekema EJ, Folea M, Kouřil R (2009) Single particle electron microscopy. Photosynth Res. doi:10.1007/s11120-009-9443-1

Chen YC, Clegg RM (2009) Fluorescence lifetime-resolved imaging. Photosynth Res. doi:10.1007/s11120-009-9458-7

Cisek R, Spencer LT, Zigmantas D, Espie GS, Barzda V (2009) Optical microscopy in photosynthesis. Photosynth Res (this issue)

Hohmann-Marriott MF, Roberson RW (2009) Exploring photosynthesis by electron tomography. Photosynth Res. doi:10.1007/ s11120-009-9452-0

Petrášek Z, Eckert H-J, Kemnitz K (2009) Wide-field photon counting fluorescence lifetime imaging microscopy: application to photosynthesizing systems. doi:10.1007/s11120-009-9444-0

Reviakine I, Bergsma-Schutter W, Brisson A (1998) Growth of protein 2-d crystals on supported planar lipid bilayers imaged in sity by AFM. J Struct Biol 121:356-362

Scheuring S, Sturgis JN (2009) Atomic force microscopy of the bacterial photosynthetic apparatus: plain pictures of an elaborate machinery. Photosynth Res. doi:10.1007/s11120-009-9413-7

Staehelin LA (1976) Reversible particle movements associated with unstacking and restacking of chloroplast membranes in vitro. J Cell Biol 71:136-158

Van As H, Scheenen T, Vergeldt FJ (2009) MRI of intact plants. Photosynth Res. doi:10.1007/s11120-009-9486-3 